teamwork and quality healthcare delivery safety'. Unprofessional behaviour impacts on patient experience and outcomes, contributes to poor teamwork and increases the risk of complaints and litigation. Most clinicians do not present problems but recurrent incidents involving the same individuals are common.

Responsibility for recognising and managing unprofessional behaviour should be encouraged at all levels of the healthcare system however clinical leaders have a particular role in both modelling professional behaviours and managing individuals and departments. Clinical leaders benefit from having policies in place for dealing with unprofessional behaviour which must have the approval and endorsement of the executive leadership team. Policies are required to be transparent, consistent and fair. Screening for health and other issues is an important early intervention in managing unprofessional behaviours. Leaders need to be aware of where to find support for doctors with health or related issues. The evidence suggests that a graduated response is most effective for managing the majority of disruptive behaviours except in the case of single egregious acts such as a sexual or physical assault.Leaders need be supported and have the necessary training to develop skills in managing unprofessional behaviours. Organisations have an obligation to provide access to human resources, legal and occupation health advice for those directly managing doctors who display unprofessional behaviours.Clinical leaders with roles in colleges, regulatory organisation and policy directorates also play an important role in creating and promoting a culture in which professionalism can thrive.

\section{LEADERSHIP STRATEGIES FOR ADDRESSING UNPROFESSIONAL BEHAVIOUR IN HEALTHCARE}

${ }^{1}$ Tweedie J*, ${ }^{2}$ Cooper J, ${ }^{3}$ Banerjee J. ${ }^{1}$ Royal College of Physicians/University College London; ${ }^{2}$ Warwick Medical School, Warwick; ${ }^{3}$ University Hospitals of Leicester, Leicester

\subsection{6/leader-2018-FMLM.73}

Unprofessional behaviour occurs when 'the use of inappropriate words, actions or inactions by a physician interferes with teamwork and quality healthcare delivery safety'. Unprofessional behaviour impacts on patient experience and outcomes, contributes to poor teamwork and increases the risk of complaints and litigation. Most clinicians do not present problems, but recurrent incidents involving the same individuals are common.

Responsibility for recognising and managing unprofessional behaviour should be encouraged at all levels of the health care system however clinical leaders have a particular role in both modelling professional behaviours and leading individuals and departments. Clinical leaders benefit from having policies in place for dealing with unprofessional conduct which must have the endorsement of the executive leadership team. Policies are required to be transparent, consistent and fair. Screening for health and other issues is an essential early intervention in managing unprofessional behaviours. Leaders need to be aware of where to find support for doctors with health or related issues. The evidence suggests that graduated response is most useful for managing the majority of disruptive behaviours except in the case of single egregious acts such as a sexual or physical assault.Leaders need to be supported and have the necessary training to develop skills in managing unprofessional behaviours. Organisations have an obligation to provide access to human resources, legal and occupation health advice for those directly leading doctors who display unprofessional behaviours.Clinical leaders with roles in colleges, regulatory organisation and policy directorates also play an essential role in creating and promoting a culture in which professionalism can thrive.

\section{Call for posters - leading innovation and improvement}

\section{GOING WITH THE FLOW: IMPROVING OUTCOMES FOR INPATIENTS WITH URGENT GASTROENTEROLOGICAL ISSUES BY OPTIMISING FOR TIME TO SPECIALTY}

John Wye*, Chantal Izquierdo, Nuala Jennings, Ehab Abdelmalek, Monica Bose. Princess Alexandra Hospital, UK

\subsection{6/leader-2018-FMLM.74}

Aims To improve outcomes of patients with emergency Gastroenterology diagnoses through optimising 'Time to Specialty' using a Plan-Do-Study-Act (PDSA) approach.

Methods This outcome measure was initially identified from personal experience of some of the authors. To justify it we collected data on patients admitted with flares of ulcerative colitis in the past year and found a strong positive correlation between length of stay (LOS) and the amount of time a patient spent outside of our specialist gastroenterology ward (Pearson correlation coefficient $=0.68$ ). We shall refer to this latter measure as Time to Specialty, our outcome measure.

We identified key moments in a patient's journey using a flow diagram and attempted to optimise them separately, measuring the effect on Time to Specialty. We identified teams involved in the admission and transfer process at each stage and engaged with them to collaboratively solve challenges. After talking to the relevant parties we created a solution that would impose a minimum of extra work as well as meeting the incentives of the different teams involved.

Results We received immediate positive feedback from the teams involved, who found that our electronic solution lifted some of the administrative burden from a pen-and-paper process. The data showed a marked improvement in the Time to Specialty for our urgent cases from 3.6 days to 0.5 days. This was matched with an improvement in average LOS from 13.9 days to 7.0 days for these urgent cases with no overall impact on LOS for the other patients.

Conclusion This project demonstrates a successful model for measuring specialty responsiveness as well as some steps that improved responsiveness in our local milieu. We hope that Time to Specialty, the time before an inpatient is taken over by the specialty team, will become a widely-used outcome measure for acute presentations where intensive specialty support is required. 